\title{
2 Theoretische Einbettung
}

\subsection{Geschichtspolitik, Gedächtnistheorie und ,Europäisierung der Erinnerung ${ }^{\circ}$}

Im Folgenden wird die Entwicklung der ,Europäisierung der Erinnerung‘ auf politischem Gebiet mit der Entstehung und dem Wandel der Konzepte ,Geschichtspolitik' und ,Gedächtnistheorie‘ in der wissenschaftlichen Literatur verknüpft. Seit Ende der 1980er Jahre der memory boom eingesetzt hat, gerät leicht aus dem Blick, dass dieser Fokus auf Erinnerung und Gedenken nicht quasi ,immer schon“ gegeben war. So verfolgten die Europäische Union bzw. ihre Vorläufer keineswegs von Anfang an eine geschichtspolitische Agenda. Bis in die 1970er Jahre lag der Fokus eindeutig auf der Ausrichtung auf die gemeinsame Zukunft und erst in den 1980ern begann man, sich auf das europäische historische Erbe zu ,besinnen'. Doch war hier zunächst ein ausschließlich positives Erbe gemeint - die Akropolis in Athen wurde etwa als erstes europäisches Kulturerbe gefördert und 1985 symbolträchtig zum Schauplatz der Vorstellung der Initiative Kulturhauptstadt Europas gemacht. (Calligaro 2015, 336) Wenn heute vielfach von der in den 1990er Jahren einsetzenden „Europäisierung des Holocaust“20 die Rede ist, so lässt dies außer Acht, dass 1993 die erste diesbezügliche Maßnahme auf europäischer Ebene (die vom Europäischen Parlament verabschiedete „Entschließung zum europäischen und internationalen Schutz der Stätten der von den Nationalsozialisten errichteten Konzentrationslager als historische Mahnmale“) die NSLager im Allgemeinen betraf und das Wort ,Holocaust“ noch gar nicht enthielt. (Kucia 2016, 102)

In diese Zeit fallen auch die ersten Gründungstexte dessen, was wir heute als ,Gedächtnistheorie‘ einerseits und als ,Geschichtspolitik‘ andererseits bezeichnen. Die Begriffe ,Erinnerungskultur‘, ,kulturelles Gedächtnis‘, ,Geschichts- und Vergangenheitspolitik' versuchten den Ende der 1980er und Anfang der 1990er sich abzeichnenden memory boom ${ }^{21}$ einzufangen, der durch die ,Universalisierung des

20 Während bereits vor den 1990er Jahren Momente wie der Eichmann-Prozess 1961 oder die Ausstrahlung der amerikanischen Fernsehserie Holocaust 1978/1979 ausgemacht werden können, in denen sich länderübergreifende Auseinandersetzungen mit dem Holocaust intensivierten, folgten die nationalen Diskussionen doch mehrheitlich eigenen Rhythmen, „die von der Rolle des jeweiligen Landes im Zweiten Weltkrieg ebenso bestimmt waren wie von aktuellen politischen Ereignissen.“ (Eckel und Moisel 2008, 13)

21 Pierre Nora spricht etwa von einer weltweiten Konjunktur des Gedächtnisses, einer Ära des leidenschaftlichen, konfliktbeladenen, fast zwanghaften Gedenkens. (Nora 2002) Das soll jedoch nicht bedeuten, dass diese „concentration of interest in the last thirty years“ nicht auf langfris- 
Holocaust“ und den Zusammenbruch des ,Ostblocks‘ weiter befeuert wurde. 1988 hatte Jan Assmann unter Rückgriff auf Maurice Halbwachs’ Studie aus 1925 das ,kollektive Gedächtnis‘ in das ,kommunikative‘ und das ,kulturelle Gedächtnis‘ unterteilt $^{22}$ und damit die Gedächtnistheorie im deutschen Sprachraum begründet, der bis heute die Arbeiten von Jan und Aleida Assmann zugrundeliegen. Schon Halbwachs hatte betont, Erinnerungen seien nie „authentisch“, sondern auf die Bedürfnisse der Gegenwart zugeschnitten: Das kollektive Gedächtnis „bewahrt nicht die Vergangenheit auf, sondern es rekonstruiert sie mit Hilfe materieller Spuren, Riten, Texte und Traditionen und mit Hilfe von neuerlichen psychischen und sozialen Gegebenheiten, das heißt mit der Gegenwart.“ (Halbwachs 1985, 296)

Ungefähr zur selben Zeit wurde im westdeutschen ,Historikerstreit‘ - 1986 ausgelöst durch Texte von Ernst Nolte, Andreas Hillgruber und Michael Stürmer sowie die Replik von Jürgen Habermas - das Wort ,Geschichtspolitik‘ zunächst als politischer Kampfbegriff und u. a. von den beiden Politikwissenschaftlern Peter Steinbach und Peter Reichel bald auch als wissenschaftliches Konzept geprägt. (Troebst 2013, 15) Während Assmann „die negativen Formen eines Vergessens durch Auslagerung, Verdrängen durch Manipulation, Zensur, Vernichtung, Umschreibung und Ersetzung“ (J. Assmann 1988, 23) in diesem kulturwissenschaftlichen Konzept nur in einem Satz erwähnte, lag der Fokus bei dem politik- und geschichtswissenschaftlichen Konzept von ,Geschichtspolitik` von Anfang an auf Deutungskämpfen:

Geschichtspolitik ist ein Handlungs- und Politikfeld, auf dem verschiedene Akteure Geschichte mit ihren spezifischen Interessen befrachten und politisch zu nutzen suchen. Sie zielt auf die Öffentlichkeit und trachtet nach legitimierenden, mobilisierenden, politisierenden, skandalisierenden, diffamierenden $u$. a. Wirkungen in der politischen Auseinandersetzung.

(Wolfrum 1999, 24f)

Der Begriff ,Vergangenheitspolitik‘ schloss bei seiner ursprünglichen, engen Bedeutung die Analyse von Identitätsstiftung zunächst nicht ein: Im Sinne einer ,justiziellen Vergangenheitsbewältigung“ führte ihn 1994 der Politikwissenschaftler Claus Offe in Bezug auf den „Neuen Osten“ ein (Offe 1994, 187), während er beim Historiker Norbert Frei 1996 mit einer anderen Konnotation erstmals auftauchte, nämlich als kritischer Ersatzbegriff für eine nicht praktizierte Vergangenheitsbewältigung in der Adenauer-Zeit der BRD. Der Politikwissenschaftler Günther Sand-

tige Entwicklungen aufbaute, wie die AutorInnen des Collective Memory Readers anhand von Grundlagentexten herausarbeiten. (Olick, Vinitzky-Seroussi und Levy 2011, 5)

22 Darunter fasst Assmann „den jeder Gesellschaft und jeder Epoche eigentümlichen Bestand an Wiedergebrauchs-Texten, -Bildern und -Riten zusammen, in deren ,Pflege' sie ihr Selbstbild stabilisiert und vermittelt, ein kollektiv geteiltes Wissen, vorzugsweise (aber nicht ausschließlich) über die Vergangenheit, auf das eine Gruppe ihr Bewusstsein von Einheit und Eigenart stützt.“ (J. Assmann 1988, 15) 
ner plädierte später in seinem zum Standardtext avancierten Beitrag über Geschichts- und Vergangenheitspolitik dafür,

als Vergangenheitspolitik den politischen, justiziellen und kulturellen Umgang einer demokratischen Gesellschaft mit ihrer diktatorischen Vergangenheit zu bezeichnen - ohne dabei symbolische Politikformen oder Diskurspolitik auszuschließen. Geschichtspolitik kann darüber hinaus - quasi als Überbegriff - die politische Instrumentalisierung von Geschichte, die Motive und Modalitäten ihrer Konstruktion, die Funktion ihrer Präsenz und politischen Virulenz in der Gegenwart in einem wesentlich umfassenderen Sinne bezeichnen. Geschichtspolitik und Vergangenheitspolitik sind folglich nicht komplementär, sondern stehen in einem hierarchischen Verhältnis zueinander.

(Sandner 2001, 7)

Doch letztlich setzte sich im deutschen Sprachraum der Begriff der Geschichtspolitik sowohl für den Umgang mit einer bestimmten Vergangenheit wie mit Geschichte im umfassenderen Sinn durch (Schmid 2009, 69) und auch im Englischen trete Stefan Troebst zufolge politics of history den Siegeszug an. (Troebst 2013, 16) „Geschichtspolitik sollen jene Diskurse und Handlungen heißen, mit denen die Deutung von Geschichte als gegenwärtige öffentliche Repräsentation einer kollektiv relevanten Vergangenheit zu politischen Zwecken betrieben wird." (Schmid 2008a, 78) Ihre Grundfunktionen seien „die Schaffung einer Tradition, die Prägung einer kollektiven Identität und die Generierung politisch-historischer Legitimität.“ (Schmid 2008a, 78) Geschichtspolitische Ansätze verstehen den Umgang mit Geschichte vor dem Hintergrund der Bedürfnisse der Gegenwart, denn verschiedene Gruppen haben nicht nur abweichende, sondern miteinander konkurrierende Erinnerungen:

Der politische beziehungsweise gesellschaftliche Konflikt besteht jedoch nicht in der ,logischen' oder ,natürlichen` Widersprüchlichkeit der Bilder und Gedächtnisse, sondern eher darin, dass die einzelnen, gruppenspezifischen [...] Formen des kollektiven Gedächtnisses sich als die Geschichte inszenieren und präsentieren, [...] was notwendigerweise zu symbolischer und politischer Marginalisierung und Ausdrängung von anderen Erinnerungen und Gedächtnissen führt.

(Niedermüller 2004, 16)

Der Politikwissenschaftler Helmut König betont, „dass das Gedächtnis in der Politik seit jeher ein heftig umkämpftes Terrain ist, zu dessen Eroberung und Besetzung von den Akteuren eine ganze Fülle von Strategien entwickelt wird. Zur Analyse politischer Systeme und politischen Handelns gehört deswegen die Gedächtnisdimension unabdingbar hinzu.“ (König 2008, 14) In der politikwissenschaftlichen Auseinandersetzung mit Geschichtspolitik liegt die Betonung tendenziell weniger als in der Geschichtswissenschaft auf der Geschichte, mit der Politik gemacht wird, als vielmehr auf den Diskursen und der Politik selbst sowie den relevanten nationalen und transnationalen AkteurInnen. 
Während also Gedächtnistheorie und das Konzept der Geschichtspolitik den memory boom zu fassen versuchten, erreichte dieser auf europäischer Ebene einen ersten Höhepunkt. Für die ,Europäisierung ${ }^{23}$ der Erinnerung، war das entscheidende Jahr 1995, in dem sich das Kriegsende 1945 zum 50. Mal jährte. Diese ,Europäisierung der Erinnerung‘ kann verstanden werden als „Prozess der relativierenden Transformation der nationalen Perspektiven, als strukturelle Veränderung und Angleichung der Geschichtsbilder im Sinne einer Anreicherung mit europäischen Bezügen.“ (Schmid 2008b, 178) Zunächst sprach bei der ersten europäischen Gedenkveranstaltung im Februar 1995 der Präsident des Europäischen Parlaments Klaus Hänsch - ein deutscher Sozialdemokrat und schlagender Burschenschafter, dessen Familie 1945 aus Schlesien geflohen war - anlässlich des Bombardements von Dresden davon, dass das Leiden deutscher Zivilisten mit jenem der Bevölkerung von Coventry, Leningrad oder Rotterdam zu vergleichen sei. (Calligaro 2015, 338) Doch Mitte des Jahres setzte im europäischen Parlament eine Entwicklung ein, die zu Beginn des neuen Millenniums ihren Höhepunkt erreichen sollte: die „Europäisierung des Holocaust“ ${ }^{24}$, verstanden als „the process of construction, institutionalization, and diffusion of beliefs regarding the Holocaust as well as formal and informal norms and rules regarding Holocaust remembrance and education that have been first defined and consolidated at a European level and then incorporated into the practices of European countries." (Kucia 2016, 100) Pakier und Wawrzyniak $(2015,4)$ sprechen in diesem Zusammenhang im Sinne der Verknüpfung von politischen und wissenschaftlichen Entwicklungen

23 ,Europäisierung“ im Allgemeinen meint hier den „Prozess des europäisch Machens“: staatliche, gesellschaftliche und individuelle AkteurInnen „reagieren keinesfalls nur auf kulturellen Anpassungsdruck, der von der politischen und ökonomischen Integration der EU ausgeht. Vielmehr agieren sie selbst und treiben Europäisierungsprozesse an, modifizieren oder blockieren diese.“ (Kaiser, Krankenhagen und Poehls 2015, 13)

24 Zunächst meint dies die Erkenntnis, dass der Holocaust auch andere europäische Staaten als Deutschland betrifft: „Dadurch, dass in den vergangenen Jahren Stück für Stück aufgedeckt wurde, dass hinter der deutschen Vernichtungsmaschinerie ein Kranz europäischer Staaten zumindest ein Teil ihrer politischen Eliten - gewissermaßen arbeitsteilig an der Diskriminierung, Ausplünderung und Ermordung der europäischen Juden beteiligt war, dient Deutschland nicht mehr als ausschließliche Projektionsfläche für die Verantwortung für den Holocaust.“ (Probst 2003, 230) Meilensteine hierfür waren die Diskussion um die Rolle der Schweizer Banken bei der ,Verwaltung des Nazi-Golds, die Debatte um die Kollaborationspolitik etwa des Vichy-Regimes in Frankreich, die Schadenersatzforderungen für Zwangsarbeit und Enteignung gegenüber verschiedenen Unternehmen und Banken in Europa sowie die erst jetzt ins öffentliche Bewusstsein getretenen Informationen über die eigenständige Organisation antisemitischer Pogrome und Morde, etwa in einigen polnischen Gegenden (Stichwort Jedwabne). 
gar von „marriage of ,memory studies“ with the European identity debate“. Die ,Europäisierung des Holocaust‘

gathered momentum between 1995 and 2000, when the EP adopted six more of its twelve Holocaust-related documents: (1) the „Resolution on a day to commemorate the Holocaust“ of 15 June 1995, (2) the „Resolution on the return of plundered property to Jewish communities“ of 14 December 1995, (3) the „Resolution on Auschwitz“ of 18 April 1996, (4) the „Resolution on restitution of the possessions of Holocaust victims" of 16 July 1998, (5) the „Resolution on countering racism and xenophobia in the European Union“ of 16 March 2000, and (6) the „Declaration on the remembrance of the Holocaust“ of 7 June 2000.

(Kucia 2016, 103)

Offenbar war 1995 die Zeit für ein gemeinsames Holocaustgedenken auf EU-Ebene noch nicht gekommen, denn es sollte bis 2000 dauern, bis sich die Idee eines europaweiten Gedenktags durchsetzte. In der zweiten Hälfte der 1990er Jahre lag der Schwerpunkt noch auf ,harten' Fragen von Entschädigung und der Restitution von Raubkunst, Raubgold usw., etwa bei der Londoner Konferenz über Nazi-Raubgold 1997 und der Washingtoner Konferenz über geraubte Vermögenswerte 1998. In diesen Kontext fällt auch die einzige explizite Aufforderung der EU an die ostmittelund südosteuropäischen Beitrittskandidaten: Im Dezember 1995 verabschiedete das Europäische Parlament die „Resolution on the return of plundered property to Jewish communities“ und forderte darin „all countries of Central and Eastern Europe which have not already done so to adopt appropriate legislation regarding the return of plundered property so that the property of Jewish communities may be returned to Jewish institutions, in accordance with the principles of justice and morality“. (European Parliament 1995)

Zeitgleich mit dem Skandal um das Schweizer Raubgold wurde 1998 in Schweden das ExpertInnennetzwerk Task Force for International Cooperation on Holocaust Education, Remembrance, and Research (ITF) gegründet, dem heute - umbenannt in International Holocaust Remembrance Alliance (IHRA) - 32 Länder angehören. Der Politikwissenschaftler Jens Kroh vermutet, die Erwähnung Schwedens im Zusammenhang mit geraubten Vermögenswerten im Eizenstat-Bericht ein Monat vor der Ankündigung einer schwedischen Informationskampagne zum Thema Holocaust spiele eine Rolle, und die Motive für die Gründung der ITF seien hauptsächlich in der Abwendung eines Image-Schadens vergleichbar jenem der Schweiz zu suchen. Als Hinweis darauf nennt er die Tatsache, dass sich Schweden bei der Gründung der ITF zunächst nur an Großbritannien und die USA - die beiden Länder, in denen die oben erwähnten Konferenzen stattgefunden hatten mit der Aufforderung um Zusammenarbeit wandte, während Israel und Deutschland erst einige Monate später, und zwar (symbolisch aufgeladen) beide gleichzeitig, in die ITF aufgenommen wurden. (Kroh 2008, 160) Welche Motive auch immer bei der schwedischen Initiative, die den Fokus von der Entschädigungs- 
auf symbolische Politik verschob, eine Rolle spielten, die Gründung der ITF und ihre enorme Aufwertung bei der Holocaust-Konferenz in Stockholm im Jahr 2000 läuteten jedenfalls eine Verlagerung von Fragen der Enteignung und geraubten Vermögens hin zu Holocaust-Education und symbolischen Erinnerungsakten. ${ }^{25}$

Das in Stockholm im Jahr 2000 abgehaltene „International Forum on the Holocaust“ war ein Schlüsselereignis für die ,Universalisierung des Holocaust”. (Allwork 2015) Erstmals nahmen hochrangige PolitikerInnen, PräsidentInnen und RegierungschefInnen, renommierte WissenschaftlerInnen, GedenkstättenmitarbeiterInnen und ZeitzeugInnen aus 46 Ländern an einer Konferenz zum Thema Holocaust teil. (Eckel und Moisel 2008, 9) Als Ergebnis wurde eine Deklaration verabschiedet, in der zwar auch andere Opfer des Nationalsozialismus als solche anerkannt wurden, der Begriff ,Holocaust‘ jedoch der Vernichtung der europäischen Jüdinnen und Juden vorbehalten blieb. Darüber hinaus verdeutlicht bereits das Datum der Veranstaltung den hohen Wert symbolischer Erinnerungspolitik: Die Konferenz fand nicht nur in den ersten Tagen des neuen Jahrtausends, sondern auch rund um den Jahrestag der Befreiung von Auschwitz statt. Die in Stockholm verabschiedete Deklaration resultierte in der Empfehlung, alle Staaten sollten diesen Tag, den 27. Januar, oder ein anderes, national bedeutsames Datum als Holocaust-Gedenktag einführen.

Auf wissenschaftlicher Ebene fand diese Entwicklung ihre Entsprechung einerseits in Deutschland in der Forderung nach einem ,negativen Gedächtnis“ in kritischer Auseinandersetzung mit den „politics of regret“ (Olick 2007) als leere Hülse von Gedenkritualen, andererseits in der These von der „Kosmopolitisierung des Holocaust“, wie sie in dem vielzitierten Werk Erinnerungen im globalen Zeitalter: Der Holocaust von Daniel Levy und Natan Sznaider aus 2001 vertreten wurde. In Formen und Traditionen des negativen Gedächtnisses führt Reinhart Koselleck 2002 (27) aus: „Die Täterschaft und ihre Taten müssen in die Erinnerung einbezogen und nicht nur die Opfer als solche und allein erinnert werden.“ Charakteristisch für dieses historische Erinnern sei Volkhard Knigge zufolge,

dass Schuld und Verantwortung nicht mehr verleugnet, abgeschoben oder überdeckt werden, sondern dass sie zu Anlässen kritischer gesellschaftlicher Selbstreflexion und Selbstvergewisserung gemacht werden. [...] Der Rückbezug auf die negative Vergangenheit muss umfassend und konkret sein, muss Opfer, Täter und gesellschaftliche wie individuelle Tatvoraussetzungen einschließen; er darf auch Ambivalenzen und Grauzonen, die sich eindeutigen Opfer-Täter-Schemata widersetzen, nicht ausweichen. Zweitens schließt kritische Selbstreflexion Pietät, die den Opfern als Opfern gilt, ein. Historisches Erinnern als Akt der Pietät ernst genommen, steht gegen alle Formen der Funktionalisierung des

25 Max Czollek $(2020,33)$ bezeichnet diese Politik zugespitzt formuliert gar als „Gedächtnistheater“. 
Erinnerns. Drittens darf Pietät aber nicht gegen Wissen und geschichtswissenschaftlich fundiertes Begreifenwollen, gegen Reflexion ausgespielt werden. Denn selbstkritisches historisches Erinnern geht in oberflächlicher moralischer Verwerfung nicht auf.

(Knigge 2008, 157-159)

Diese ,heiklen' Fragen wurden zunächst nur in Bezug auf NS-Deutschland gestellt, im Zuge der ,Europäisierung des Holocaust' dann aber auch in Bezug auf die Kollaborationsregime. ,Europäisierung der Erinnerung' bedeutete bis zur EU-Osterweiterung ,Europäisierung der Holocaust-Erinnerung‘: Der Holocaust wurde als negativer Gründungsmythos Europas (Judt 2006) begriffen. ${ }^{26}$

\subsection{Universalisierung oder Kosmopolitisierung des Holocaust}

Diese europäische Entwicklung lässt sich nur im Kontext der von den USA ausgehenden ,Universalisierung des Holocaust` begreifen bzw. dessen, was die Soziologen Daniel Levy und Natan Sznaider zunächst positiv konnotiert als „Kosmopolitisierung des Holocaust“ bezeichneten. Die hier vorgenommene verschränkende Periodisierung politischer und wissenschaftlicher Entwicklungen erlaubt es uns, das Werk von Levy und Sznaider als ,Kind seiner Zeit', der zu diesem Zeitpunkt noch kaum hinterfragten ,Universalisierung des Holocaust', einzuordnen. Die beiden bewerten das „Zeitalter der Globalisierung“ optimistisch und sehen einen Wandel von nationalen zu kosmopolitischen Erinnerungskulturen auch im Sinne einer „Kampfansage an die versteinerte Wissenschaft, die nicht imstande ist, sich vom Nationalstaat zu verabschieden.“ (Levy und Sznaider 2001, 9) Die Erinnerung an den Holocaust mache die „Katastrophe Europas zum Ausgangspunkt neuer grenzüberschreitender Solidarität“, denn der Umgang mit dem Holocaust öffne das Verständnis für neue Erinnerungskulturen in der ,Zweiten Moderne'. Die Erinnerung an den Holocaust werde in einer Epoche ideologischer Ungewissheiten zu einem Maßstab für humanistische und universalistische Identifikationen. (Levy und Sznaider 2001, 10) Dadurch würden national übergreifende Gedenkkulturen zur Grundlage für eine globale Menschenrechtspolitik. (Levy und Sznaider 2001, 11) Paradoxerweise trage gerade das Unvorstellbare des Holocaust zu seiner Entkontextualisierung bei, was Levy und Sznaider positiv bewerten - als Modell für Gut und Böse, Schuld und Unschuld. (Levy und Sznaider 2001, 150) Die so neu entstandene „Schicksalsgemeinschaft“ definiere sich nicht mehr durch nationale Erfahrungen, sondern nehme den Holocaust

26 Zur Kritik an der Vorstellung einer ,Stunde Null‘ nach 1945, die Kontinuitäten ausblendet, siehe Kaiser 2015, 369; Kaiser, Krankenhagen und Poehls 2012, 138. 
zum Anlass, neue gemeinsame Bezüge jenseits des Nationalstaats herzustellen. (Levy und Sznaider 2001, 14) „Die neuen Erinnerungen gelten den Opfern und nicht den Tätern oder Helden. Damit ist der Holocaust zu einem universalen ,Container für Erinnerungen an unterschiedliche Opfer geworden." (Levy und Sznaider 2001, 223) ${ }^{27}$

2002 diagnostizierte auch der amerikanische Soziologe Jeffrey C. Alexander, dass der Holocaust zu einem universalisierten Symbol geworden sei. Doch diese ,Universalisierung' habe zwei sehr verschiedene Gesichter. Einerseits lässt sich mit Alexander $(2002,6)$ festhalten: „the originating historical event, traumatic in the extreme for a delimited particular group, has come over the last fifty years to be redefined as a traumatic event for all of humankind“. Er bezieht sich dabei unter anderem auf Dan Diners (1988) Begriff „Zivilisationsbruch Auschwitz“. Zugleich hält Alexander $(2002,51)$ aber auch die andere Bedeutung der ,Universalisierung' fest:

Evoking the Holocaust to measure the evil of a non-Holocaust event is nothing more, and nothing less, than to employ a powerful bridging metaphor to make sense of social life. The effort to qualify as the referent of this metaphor is bound to entail sharp social conflict, and in this sense social relativization, for successful metaphorical embodiment brings to a party legitimacy and resources.

Universalisierung kann also sowohl heißen, den Holocaust als ,Zivilisationsbruch anzuerkennen, als auch, dass verschiedene Opfergruppen explizit oder implizit betonen, sie hätten ,wie die Juden' (Eder 2017, 18; Rothberg 2009; Miller 2010; MacDonald 2002) gelitten. Während der Begriff ,Kosmopolitisierung des Holocaust‘ die positive Bewertung dieser Entwicklung enthält, ist ,Universalisierung (Eckel und Moisel 2008; A. Assmann 2010, 99; Leggewie und Lang 2011, 24) der neutralere Begriff, mit dem sich das Phänomen in seinen verschiedenen Ausprägungen erfassen lässt, ohne die Entwicklung normativ aufzuladen.

Auch Levy und Sznaider bessern gewissermaßen im Vorwort zur Neuauflage ihres Buches fünf Jahre später ihre zunächst ungebrochen positive Einschätzung nach, wenn sie nun kritischer schreiben:

Die Erinnerung an den Holocaust wird zu einer europäischen Erinnerung, die Europa dazu verhelfen kann, ein eigenes (wenn auch negatives) Wertesystem zu entwickeln. Der Preis, der dafür gezahlt wird, ist die Entkontextualisierung der Geschichte. Wenn aus ehemaligen Feinden nun Freunde werden, muss der alte historische Kontext verdrängt werden.

(Levy und Sznaider 2007, 11)

27 Zur Doppelbedeutung des deutschen Wortes ,Opfer‘ als ,sacrificium‘ und ,victima‘ siehe Assmann 2006, 73. 
Der Preis der Entkontextualisierung sei, dass es „in diesem Diskurs keine Juden und keine Deutschen mehr geben darf. Es gibt nur Menschen und Menschheit, wie auch aus dem Begriff der ,Verbrechen gegen die Menschheit' und der Entstehung eines moralischen und legalen Kodex gegen ,Völkermord“ hervorgeht.“ (Levy und Sznaider 2007, 12f) Auch in einem neueren Buch kritisiert Sznaider: „Das jüdische Gedächtnis ist aus dem europäischen Diskurs verschwunden. Und das trotz aller Rituale und Gedenktage! Oder vielleicht auch deswegen." (Sznaider 2008, 7) Laut Dan Diner tritt an die Stelle des verloren gegangenen historischen Urteilsvermögens „ein universell drapierter moralisierender Diskurs über unterschiedslose Opferschaft. Ein solcher Diskurs wird in letzter Konsequenz vor einer Dekonstruktion des Gedächtnisses an den Zweiten Weltkrieg ebenso wenig halt machen wie vor der Geltung und Bedeutung des Holocaust.“ (Diner 2007, 8f) Diese ,unterschiedslose Opferschaft“ wird auch in Deutschland, dem Land des ,negativen Gedächtnisses‘, in Bezug auf Bombenopfer und Vertriebene konstruiert. (Salzborn 2007) Der französische Zeithistoriker Henry Rousso diagnostiziert im Zusammenhang damit einen sich beschleunigenden Prozess der „Viktimisierung“ bzw. der „Geschichtsbetrachtung aus der Opferperspektive“ nach der Wende vom 20. zum 21. Jahrhundert:

Es ist erstaunlich, wie sehr historische Erfahrungen wie der Widerstand gegen den Nationalsozialismus oder die antikolonialen Befreiungskämpfe heute den Opferstatus hervorkehren, während sie in der Vergangenheit eher die Figur des Helden bevorzugten (des Märtyrers, der für eine Sache stirbt und sich für die Gemeinschaft opfert). Es handelt sich hier um den bedeutsamen Übergang von einem politischen zu einem moralischen Muster der Vergangenheitsbetrachtung. [...] Allerdings hat die Identifikation mit den Opfern, die in der traditionellen Geschichtsbetrachtung der Staaten, Sieger, Gelehrten usw. ja tatsächlich vergessen wurden, heute zur Überbewertung dieser Perspektive geführt.

(Rousso 2004, 374)

Diese Entkontextualisierung der historischen Ereignisse im Zuge der „Universalisierung des Holocaust“ (Eckel und Moisel 2008) als „negative Ikone“ (Diner 2007, 7) der Menschheit und „Container“ für andere Erinnerungen ist nur eines der Probleme, die spätere wissenschaftliche Werke thematisieren. Ein weiteres ist die Appropriation einer transnationalen Holocausterinnerung für die jeweiligen eigenen nationalen Zwecke. (Niven und Williams 2020, 143)

\subsection{Die ,Osterweiterung` der Europäisierung der Erinnerung}

Das Verständnis des Holocaust als einzigartiges Ereignis führt zu einem Konflikt mit anderen Opfererfahrungen. In Kontext dieser Untersuchung ist vor allem die Frage relevant, welche Auswirkungen die ,Europäisierung des Holocaust‘ als ne- 
gativer Gründungsmythos Europas (Leggewie und Lang 2011, 15; Uhl 2007) auf die EU-Beitrittsbemühungen der postsozialistischen Länder hatte. Während amerikanische PolitikerInnen in Bezug auf den NATO-Beitritt auf die Bedeutung der ITF hinwiesen, um sich gemeinsamer Werte und Ziele der NATO-Beitrittskandidaten $\mathrm{zu}$ versichern, ${ }^{28}$ finden sich keine Belege dafür, dass EU-Gremien einen bestimmten Umgang mit dem Holocaust offiziell zum Beitrittskriterium gemacht hätten. Die meisten Beitrittskandidaten traten aber vor oder rund um ihren EU-Beitritt auch der ITF bei, die sich 2013 in International Holocaust Remembrance Alliance (IHRA) umbenannte. ${ }^{29}$ Genau dieser Prozess der zunächst scheinbar direkten Übernahme des westeuropäischen Umgangs mit dem Holocaust wird unten am Beispiel der Museen beleuchtet.

In den prominentesten wissenschaftlichen Publikationen zur Universalisierung und Europäisierung des Holocaust bleibt der Punkt sehr schwammig, inwiefern diese Entwicklung auch auf ,Osteuropa‘ zutraf. (Olick 2015, x) So schränken Levy und Sznaider an einer Stelle ein, der Holocaust sei „in vielen westlichen Staaten zum moralischen Maßstab der Unterscheidung zwischen gut und böse geworden“ (Levy und Sznaider 2001, 15), nur um später wieder zu behaupten, die „Kosmopolitisierung des Holocaust“ bzw. der Erinnerung daran habe ihn zu einem „global gültigen Wert“ (Levy und Sznaider 2001, 150) gemacht. Tony Judt schreibt zwar, für die „Osteuropäer“ sei die Tatsache, dass sich der Westen am Ende des Jahrhunderts so intensiv mit dem Holocaust beschäftigte, „äußerst verstörend“ (Judt 2006, 955) gewesen, denn Europa möge zwar geeint sein, die europäische Erinnerung bleibe jedoch asymmetrisch. Doch letztlich seien das bloß „Schatten“ (Judt 2006, 961), denn „das neue Europa, durch die Zeichen und Symbole seiner schrecklichen Vergangenheit zusammengeschlossen, ist eine bemerkenswerte Leistung.“ (Judt 2006, 966) „Jeder, der zu Beginn des 21. Jahrhunderts wirklich Europäer werden will, [muss] zunächst ein neues und weit bedrückenderes Erbe auf sich nehmen“. Heute sei „die einschlägige europäische Bezugsgröße [...] die Vernichtung. Die Anerkennung des Holocaust ist zur europäischen Eintrittskarte geworden“ (933), so Judt, ohne diese Konditionalität zu problematisieren. Der Politikwissenschaftler Claus Leggewie begreift hingegen den Holocaust als „Kern des europäischen Geschichtsbewussteins“: „Darum ranken sich konzen-

28 So der mit dem Holocaust-Gedenken Beauftragte im US-Außenministerium 2003 (zit. n. Kroh 2008, 163).

29 Ausnahmen waren mit Estland (2007 beigetreten) und Slowenien (2011 beigetreten) die beiden Länder, die nur eine sehr kleine jüdische Bevölkerung hatten, sowie das erst 2018 beigetretene Bulgarien, das lange von sich behauptete, ,seine“ jüdische Bevölkerung gerettet zu haben, ohne die Ermordeten aus den ,neuen bulgarischen Gebieten` Mazedonien und Thrakien $\mathrm{zu}$ berücksichtigen. 
trisch weitere sechs, zunehmend strittige Kreise europäischer Erinnerung, teils in Assimilation an den, teils in Distanz oder Indifferenz gegenüber dem Kern“ (Leggewie und Lang 2011, 12), wobei die Erinnerung an den Gulag und die sowjetischen Verbrechen den zweiten Kreis bildet. Er warnt allerdings vor „grobschlächtigen Varianten der Totalitarismusthese und fordert, „das ,Singuläre“ am Zivilisationsbruch der industriell-bürokratischen Vernichtung der europäischen Juden herauszustellen, ohne die systematische Ausrottung der ,Klassen- und Volksfeinde“ im sowjetischen Machtbereich herunterzuspielen.“ (Leggewie und Lang 2011, 25)

Anlässlich des 60. Jahrestags des Kriegsendes wurden auf EU-Ebene die letzten Deklarationen verabschiedet, die noch als ungebrochener Ausdruck der ,Europäisierung des Holocaust‘ gedeutet werden können. Die wichtigste von ihnen war die „Entschließung des Europäischen Parlaments zum Gedenken an den Holocaust sowie zu Antisemitismus und Rassismus“, die den „27. Januar in der gesamten Europäischen Union zum Europäischen Holocaustgedenktag“ erklärte und die Holocaust-Erinnerung mit der Bekämpfung des heutigen Rassismus und Antisemitismus verknüpfte. (Europäisches Parlament 2005) Auch wenn dieser Fokus auf den Holocaust niemals unwidersprochen war, wurden bei den Feierlichkeiten zum 9. Mai 2005 in Moskau doch erstmals die Gräben unübersehbar: Stefan Troebst zufolge standen sich hier

das postsowjetische Russland auf der einen Seite und etliche mittlerweile zur EU gehörigen ehemaligen Satelliten bzw. Republiken der UdSSR, zuvörderst Polen, Litauen, Lettland und Estland, auf der anderen gegenüber - bei deutlicher Parteinahme der USA für die ostmitteleuropäische Sicht auf die Geschichte sowie bei vorsichtiger Unterstützung Deutschlands für die russländische Interpretation. Aus Moskauer Sicht [...] steht der 9. Mai für den „Sieg über den Faschismus“ und für die „Befreiung Europas“. Aus ostmitteleuropäischer, hier vor allem polnischer Perspektive indes fällt die Zurückdrängung der deutschen Besatzungsmacht durch die Rote Armee im Sommer 1944 mit dem Imstichlassen der Warschauer Aufständischen, der Errichtung einer kommunistischen Diktatur unter sowjetischem Kuratel sowie schließlich mit dem „Verrat“ der anglo-amerikanischen Verbündeten Polens im Februar 1945 in Jalta zusammen. (Troebst 2006, 24)

Damit scheint auf der Ebene der EP-Resolutionen eine Erwähnung des Holocaust ohne gleichzeitigen Verweis auf sowjetische Verbrechen nicht mehr möglich, denn in der „European Parliament resolution on the $60^{\text {th }}$ anniversary of the end of the Second World War in Europe on 8 May 1945“ wird hervorgehoben, dass „for some nations the end of World War II meant renewed tyranny inflicted by the Stalinist Soviet Union“ (Kucia 2016, 106). Es folgen 2008 die „Erklärung des 23. August zum Europäischen Tag des Gedenkens an die Opfer von Stalinismus und Nazismus“ und 2009 die „Entschließung des Europäischen Parlaments zum Gewissen Europas und zum Totalitarismus“, deren Namen bereits einen klaren Hinweis auf die veränderten Begrifflichkeiten nach der großen EU-Osterweiterung 2004 geben. 
Die Idee, den 23. August, den Jahrestag des Hitler-Stalin- respektive MolotowRibbentrop-Pakts 1939 zu einem europäischen Gedenktag zu erklären, entstand 2008 während der slowenischen EU-Ratspräsidentschaft und wurde in Vorbereitung auf die tschechische 2009 weiterentwickelt. Die tschechische Regierung finanzierte 2008 eine am staatlichen Institut für die Erforschung totalitärer Regime in Prag ausgerichtete Konferenz über European Conscience and Communism, im Rahmen derer die Prager Deklaration verabschiedet wurde. Auf ihrer Grundlage unterzeichneten am 23. September 2008409 Mitglieder des Europäischen Parlaments eine Erklärung zur Unterstützung der Errichtung eines „European Day of Remembrance for Victims of Stalinism and Nazism“. Die Unterstützungserklärung aus 2008 betonte Aline Sierp zufolge

the similarities between the Nazi and the Communist regime and calls for equal treatment of victims of both ideologies. [...] An analysis of the document texts shows clearly that the already well-institutionalised remembrance of the Nazi crimes served as a model for the remembrance of Communist crimes but that it was also understood as a competitor within an allencompassing European heritage.

(Sierp 2017, 447)

Sierp beschreibt die heftigen Kämpfe, die vor allem die sozialistische Fraktion des Europäischen Parlaments gegen die Initiative der Europäischen Volkspartei bis zur Verabschiedung der Resolution im April 2009 unter dem stark veränderten Namen „Entschließung des Europäischen Parlaments zum Gewissen Europas und zum Totalitarismus“ führte, die mit $553 \mathrm{zu} 44$ angenommen wurde. Die Einsetzung einer von den Europäischen SozialistInnen angeführten Geschichtsarbeitsgruppe gegen eine Umschreibung der Geschichte und gegen die Gleichsetzung der sowjetischen mit den NS-Verbrechen im Vorfeld der Entschließung habe wesentlich dazu beigetragen, dass diese „distinguishes Stalinism clearly from Communism, includes also Southern European dictatorships in the list of totalitarian regimes, and explicitly mentions the uniqueness of the Holocaust.“ (Sierp 2017, 448) Zwischen 2009 und 2011, als westliche Mitgliedsländer die EURatspräsidentschaft innehatten, veränderte sich nicht viel und der 23. August setzte sich nicht als Europäischer Gedenktag durch. Erst als 2011 mit Polen wieder ein ostmitteleuropäisches Land die Ratspräsidentschaft innehatte, verabschiedete der Rat die „Warsaw Declaration on the occasion of the European Day of Remembrance for Victims of Totalitarian Regimes“ und riet darin den Mitgliedstaaten, den 23. August als „European Day of Remembrance for the Victims of all Totalitarian Regimes“ zu begehen. (Sierp 2017, 449)

Ausdruck der Bemühungen um eine Inklusion der postsozialistischen Perspektive in transnationale Erinnerungsprozesse sind auch das 2005 ins Leben gerufene und seit 2008 operierende Europäische Netzwerk Erinnerung und Solidarität (ENRS) mit Sitz in Warschau und die ebenfalls zunächst am Institut für die Erfor- 
schung totalitärer Regime in Prag angesiedelte Platform of European Memory and Conscience, ein unmittelbares Ergebnis der „Entschließung des Europäischen Parlaments zum Gewissen Europas und zum Totalitarismus“ aus 2009. Büttner und Delius zufolge sei das von den Kulturministerien Deutschlands, Polens, Ungarns und der Slowakei gegründete ENRS stark von der wissenschaftlichen Bemühung um Anerkennung unterschiedlicher erinnerungskultureller Perspektiven geprägt. In ihrem Mission Statement werfen sie folgende Fragen auf: „How can we create new, broader and internally more differentiated narratives? How can we shape the memory of the victims of National Socialism and communism without erasing their differences and without allowing anyone to compete over victim counts or to trivialise the discussion?" ${ }^{30}$ Die Plattform hingegen

actively pursues an agenda of establishing new ,anti-totalitarian' narratives and, more concretely, strives for the adoption of transitional justice measures regarding crimes committed by Communist regimes on a European scale. Thus, contrary to its name, the Platform acts as a classic lobby actor in European memory politics rather than as an open ,Platform‘ of debate on ,European Memory and Conscience‘.

(Büttner und Delius 2015, 401)

Eine von der Plattform erstellte Ausstellung heißt folglich Totalitarianism in Europe, das Schul-Lesebuch Lest We Forget. Memory of Totalitarianism in Europe (Purves 2013) enthält die Lebensgeschichten von 30 von „Totalitarismus“ betroffenen Menschen aus 16 Ländern. Mitglieder der Plattform sind unter anderem auch einige der hier untersuchten Museen bzw. die jeweilige übergeordnete Stiftung: das Haus des Terrors, das Museum der Okkupation Lettlands, das Genozid- und Widerstands-Forschungszentrum Litauens (die Trägerorganisation des Museums der Genozidopfer in Vilnius) sowie das Museum des Warschauer Aufstands. 2014 wurde das Institut für die Erforschung totalitärer Regime aus der Plattform ausgeschlossen und das Sekretariat zog von Prag nach Brüssel um. Als vielsagenden Grund für diesen radikalen Bruch mit dem Prager Institut gab der Leiter der Plattform an: „the Institute has been infiltrated by Communist collaborators“. (Platform 2014) Die neue tschechische sozialdemokratisch angeführte Regierungskoalition von Bohuslav Sobotka hatte einen neuen Direktor des Instituts eingesetzt, dessen neuer Kurs sich nicht mit den totalitarismustheoretischen Prämissen der Plattform vertrug.

Małgorzata Pakier und Joanna Wawrzyniak resümieren bezüglich der Veränderung der europäischen Erinnerungslandschaft:

30 Text der alten Webseite (http://www.enrs.eu/en/goals), abgerufen am 4.5.2017, heute nicht mehr abrufbar. 
While previously the East Europeans found it difficult to draw the attention of their Western counterparts with regard to questions of their history and memory, the official commemorations and public controversies of the last few years show that Eastern Europe has become an important trigger for discussions about the content and form of a European narrative.

(Pakier und Wawrzyniak 2015, 1f)

Es kann festgehalten werden, dass es den postsozialistischen Mitgliedstaaten unmittelbar nach dem EU-Beitritt gelungen ist, den Fokus von der ,Europäisierung des Holocaust' auf eine ,Europäisierung der Erinnerung' zu verschieben, die auch die Erinnerung an den Stalinismus und Staatssozialismus und ihre Verbrechen enthält. Diese wird aber nicht einfach als eine weitere unverzichtbare Komponente in den europäischen Kanon hereingeholt. Vielmehr befördern die neuen postsozialistischen EU-Mitgliedsländer eine Gleichsetzung: „since the enlargement, another memorial frame has become increasingly institutionalized at the EU level: the description of ,Nazism and Stalinism as equally evil“". (Calligaro 2015, 339)

Der 23. August lässt sich dabei mit Heidemarie Uhl als Antithese zum 27. Januar begreifen. Denn während die ,Europäisierung des Holocaust‘ und das Gedenken an die Befreiung von Auschwitz als Ausdruck dessen verstanden werden können, was oben als ,negatives Gedächtnis', als Frage nach der Involvierung der eigenen Gesellschaft in die NS-Verbrechen bezeichnet wurde, befördert der 23. August dieses negative Erinnern nicht: „In der Erinnerungskultur der Post-1989-Gesellschaften ist das ,eigene Volk' ein unschuldiges Opfer grausamer Unterdrückung von außen, die Involvierung der eigenen Gesellschaft in das kommunistische Herrschaftssystem kann so externalisiert werden“ (Uhl 2009) - wie auch die NS-Kollaboration, ließe sich hier ergänzen.

Wenn in dieser Arbeit untersucht wird, wie sich die ,Europäisierung der Erinnerung' in den mit der Zeit des Zweiten Weltkriegs befassten Museen ausgewirkt hat, soll es keineswegs darum gehen, ,Geschichtsklitterung، aus einer ,akkusatorisch-moralischen' Position heraus zu untersuchen, sondern es werden ,gerade die widersprüchlichen Geschichtsbilder, Tabuisierungen und Schweigestellen“ (Gerbel et al. 2005, 20) zum Ausgangspunkt der Analyse gemacht. Verschiedene parteiische Vergangenheiten entstehen durch das strategische Gegenhandeln verschiedenster politischer Kräfte und werden, wenn es strategisch-taktisch nottut, revidiert oder der veränderten Situation angepasst:

„Vergangenheit“ ist das - nie einheitliche - Produkt dieses Gegenhandelns. Dabei darf nicht übersehen werden, dass dieses Produkt auch in Schweigen oder Stille bestehen kann. Dies bedeutet nicht unbedingt die Abwesenheit von Erinnerung, nicht einfach das Aussetzen der Konstruktionsleistung. Vielmehr können Leerstellen selbst das Ergebnis einer Konstruktion sein, das dem vorübergehenden Sieg einer bestimmten Konstruktion geschuldet ist, also dem Sieg einer bestimmten Vergangenheitsversion, der es gelungen 
ist, andere, alternative Versionen an den Rand (in die Stille) zu drängen, zu überschreiben und auszucanceln, sie „stillzulegen“. Stille herrscht dort, wo die „Stilllegung“ des gegnerischen Diskurses gelungen ist.

(Marchart 2016, 54)

Besonders das Vergessen und Stilllegen von Geschichte sind hier von Interesse.

\subsection{Das Museum und seine theoretische Verortung}

Im Zentrum dieser Arbeit stehen Museen, die der im Kontext der Museumstheorie vielzitierte Benedict Anderson als eine ,institution of power“ (Anderson 1996, 163) begreift, eine tragende Säule bei der Schaffung moderner nationaler Identität. Robin Ostow $(2008,3)$ fasst Museen in heutigen Demokratien als Schlüsselorte für kulturelle und Geschichtspolitik, die nun mittels neuer Medien - nicht nur, aber sehr stark auch in postsozialistischen Ländern - als High-Tech-Museen um- oder neugestaltet werden. Alison Landsberg begreift Museen als Orte einer „prosthetic memory“, anhand derer man untersuchen kann, „what it means to own or inhabit a memory of an event through which one did not live." (Landsberg 2004, 129) Jan Assmann betont, Museen mit einem gesetzlich verankerten nationalen Status komme als Orten einer symbolischen nationalen Repräsentation eine bedeutende Rolle bei der „Erfindung der Nationen“ zu. (J. Assmann 1999, 31) Minderheitenmuseen können im Gegensatz dazu durch Sichtbarmachung bisher unterdrückter Gegennarrative Ergänzungen zum Kanon bieten. (Pieper 2006, 23) Vor allem aber, wenn sie mit einem traditionell-ethnologisierenden Blick die ,Anderen' darstellen, können aber auch sie Stereotype reproduzieren. ${ }^{31}$ Museen sind in jedem Fall an der Produktion von Wissen und von Geschichte beteiligt. Sie sind „keineswegs neutrale Räume der Wissensvermittlung und -popularisierung, die zeigen, wie ,es' früher war. Vielmehr manifestieren sich im Gezeigten kulturelle Muster, Ein- und Ausschlussmechanismen und - sozialwissenschaftlich gesprochen - soziale, ethnische oder religiöse In- und Outgroups." (Sommer-Sieghart 2006, 159) Wie Geschichtspolitik im Allgemeinen sagen auch zeithistorische Museen dabei immer vor allem etwas über die Bedürfnisse der Gegenwart aus. Dies ist nicht nur im politischen Sinne gemeint, sondern zielt auch auf neue Medien, den Unterhaltungsfaktor:

Although holding fast to classical modern notions of the museum as a public educator and as a catalyst of social reform, the new museology redefines curatorial and outreach practice as extending far beyond the selection and display of instructive samples of knowledge, and

31 Als Beispiel wäre hier etwa die von Adam Bartosz in Tarnów im Rahmen des Ethnographischen Museums eingerichtete Roma-Ausstellung zu nennen. (Bartosz 1998) 
now incorporating dimensions such as entertainment, empowerment, experience, ethics, and narrative endeavour.

(Andermann und Arnold-de Simine 2012, 5)

Zum Begriff des memorial museums muss präzisiert werden, dass dieser zunächst solche Gedenkinstitutionen bezeichnete, die nicht in situ, also nicht an den Orten der Verbrechen waren - allen voran das 1993 eröffnete USHMM in Washington, D. C. und Yad Vashem in Jerusalem, das 1957 als Gedenkstätte eingerichtet wurde und dessen Holocaust History Museum 2005 seine Pforten öffnete. ${ }^{32}$ Im Zuge der „travelling memory“ (Erll 2011, 11), der oben beschriebenen ,Universalisierung“ und ,Europäisierung des Holocaust" orientierten sich aber der Zeit des Zweiten Weltkriegs und der sozialistischen Ära gewidmete, neue ständige Ausstellungen in postsozialistischen Ländern Ostmittel- und Südosteuropas stärker an diesem Konzept als an In-situ-KZ-Gedenkstätten in Deutschland und Polen, wie gezeigt werden wird. Aus diesem Grund habe ich für diese Arbeit bewusst den auf Deutsch etwas ungewohnt klingenden Begriff des ,Gedenkmuseums' als direkte Übersetzung von memorial museum gewählt, um den ,Import‘ des Memorial-Museum-Konzepts auch in postsozialistische In-situ-Museen und seine teils problematischen Folgen herausarbeiten zu können. ${ }^{33}$

Gedenkmuseen unterscheiden sich etwa von Häusern der Geschichte dadurch, dass sie einem historischen Ereignis gewidmet sind und zunächst allgemein gesagt eines Massenverbrechens gedenken. Solche memorial museums stellen Paul Williams zufolge in gewisser Weise einen inhärenten Widerspruch dar:

A memorial is seen to be, if not apolitical, at least safe in the refuge of history. [...] A history museum, by contrast, is presumed to be concerned with interpretation, contextualization, and critique. The coalescing of the two suggests that there is an increasing desire to add both a moral framework to the narration of terrible historical events and more indepth contextual explanations to commemorative acts. That so many recent memorial museums [...] find themselves instantly politicized itself reflects the uneasy conceptual coexistence of reverent remembrance and critical interpretation.

(Williams 2007, 8)

Selbstredend sind auch Mahnmale ohne Museum keinesfalls unpolitische Orte, wie die Kontroverse um das 2014 am Freiheitsplatz in Budapest errichtete Denk-

32 Als staatliche Holocaust-Museen stehen diese in einem Spannungsverhältnis zwischen „the massive might of official remembrance, and the quiet, intimate remembrance of individual survivors.“ (Stone 2004, 518)

33 So nennt sich das kroatische Museum auf dem Gelände des ehemaligen Ustaša-KZs „Jasenovac Memorial Museum“ - Memorijalni muzej (http://www.jusp-jasenovac.hr/Default.aspx? sid=6559), gibt als Vorbilder das USHMM, Yad Vashem und das Anne-Frank-Haus an und konzentriert sich vor allem auf die individuellen Opfer, während es das Gelände, auf dem es sich befindet, erstaunlich vernachlässigt. (Radonić 2014a, 499) 


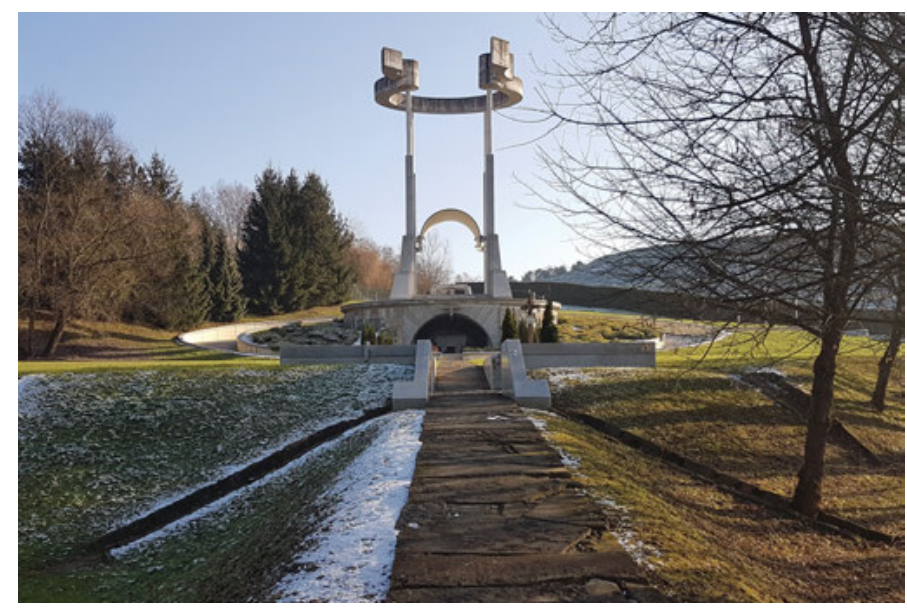

Abb. 1: Die Gedenkstätte Teharje in Slowenien.

mal für die Opfer der deutschen Besatzung zeigt. (Oláh 2016) Doch manchmal bestehen Mahnmale oder gar ganze Gedenkareale bloß aus einer künstlerischen Installation und einem Gedenkraum ohne jegliche Benennung oder textuelle Interpretation ihres Gegenstands. Ein Beispiel dafür ist etwa das größte Gedenkgelände Sloweniens, Teharje bei Celje. (Abb. 1) Dort errichtete die Wehrmacht 1943 zunächst ein Militärlager für die eigenen Truppen und sperrte dann gegen Ende des Krieges Gefangene ein, die bei der Verteidigung von Celje für ,niedere Tätigkeiten‘ eingesetzt worden waren. Nach dem Krieg 1945 internierten dann Tito-PartisanInnen dort vor allem Domobranzen, slowenische NS-Kollaborateure, und töteten Tausende von ihnen in der Umgebung des Lagers. Am Eingang des riesigen, 2004 eröffneten „Gedenkparks Teharje“ steht nur, er sei „allen hier gestorbenen und aus dieser Gegend in den Tod verschleppten“ gewidmet, nirgendwo ein Wort darüber, wer wen wann warum hier eingesperrt oder getötet hatte. Im Zeitgeschichtemuseum in Ljubljana widmet sich hingegen seit 1998 die Ausstellung The Dark Side of the Moon: A Short History of Totalitarianism in Slovenia, 1945-1990 diesen Kriegsendverbrechen.

Gedenkmuseen haben zwei Komponenten: als Orte des Totengedenkens die Erinnerung an eine bestimmte Gewaltgeschichte sowie ferner den Anspruch, mittels Adaption der Vergangenheit ,eine Bewusstseinsbasis für aktuelle gesellschaftliche und politische Probleme zu schaffen, auf der Handlungsmaximen und Werte formuliert werden.“ (Pieper 2006, 24) Ausstellungen sind Orte, an denen Signifikations- und Kommunikationsprozesse stattfinden: AusstellungskuratorInnen formulieren Inhalte, Absichten und Erwartungen, GestalterInnen übertragen sie in räumliche Arrangements, BesucherInnen machen Erfahrungen und sam- 
meln Erkenntnisse, die idealerweise mit den zu vermittelnden Inhalten übereinstimmen. (Scholze 2004, 12) Im Ausstellen kreuzen sich Deutungsabsichten von Ausstellenden, Bedeutungen des Ausgestellten und Bedeutungsvermutungen von BesucherInnen. (Muttenthaler und Wonisch 2006, 59) Der Prozess des Ausstellungsmachens bleibt den BesucherInnen verborgen, dem Resultat gehen Kämpfe um Deutungshierarchien und Kompromisse voraus, politische Einflussnahmen, wissenschaftliche und künstlerische Abwägungen. Gruppen und Themen, die in der Ausstellung verhandelt werden, erlangen Präsenz und Sichtbarkeit. Untersucht wird auch das, was ich als ,Hierarchie der Sichtbarkeit' verschiedener Opfergruppen in der Ausstellung bezeichne - der Mehrheitsbevölkerung, der jüdischen sowie der Roma-Opfer.

Gedenkmuseen befinden sich im Spannungsfeld konfligierender gesellschaftlicher Machtverhältnisse und Deutungsmuster. Sie sind Orte, an denen Identität geschaffen und offizielle Geschichtspolitik kanonisiert, an denen das zum jeweiligen Zeitpunkt ihrer Schaffung dominante historische Narrativ als Fundament der Gegenwart sichtbar gemacht wird. Sie können aber auch das hegemoniale Narrativ in Frage stellen.

In beiden Fällen ist die Entscheidung darüber, welche Objekte oder Bilder man verwendet, wie man sie organisiert und welchen Ort man für die Ausstellung wählt - ein neu errichtetes Museumsgebäude oder einen historischen Verbrechensort - von politischen, ethischen und ästhetischen Fragen geleitet. Auch hier besteht ein Spannungsverhältnis zwischen dem Anspruch einerseits, ,authentische Beweise $z u$ liefern und andererseits dem Bemühen, ein emotionales, dramatisches Erlebnis für die BesucherInnen zu kreieren. (Williams 2007, 21) Stellvertretend für andere US-amerikanische AutorInnen befürworten Spencer R. Crew und James E. Sims eine Inszenierung und eine narrative Ausstellung, denn ,the problem with things is that they are dumb. They are not eloquent, as some thinkers in art museums claim. They are dumb. And if by some ventriloquism they seem to speak, they lie." (Crew und Sims 1990, 159) Oder in den Worten des Direktors des USHMM Michael Berenbaum: „Artifacts, architecture and design are subservient to the tale that is being told. They are the midwife of the story." (Zit. n. Shenker 2015, 66) Andere, insbesondere deutschsprachige AutorInnen betonen vor allem die ,Aura“ des Objekts und kritisieren an narrativen Museen, dass diese Geschichte immer nur aus einer Perspektive erzählen können - heutzutage meist aus der des Opfers. So führt etwa der langjährige Direktor der Gedenkstätte Buchenwald aus:

Dokumentierend-argumentierende Ausstellungen, wie die in Buchenwald, sind nicht nach einem vorgehenden Narrativ - wohl aber wissenschaftlichen Erkenntnissen - geordnet, sondern verstehen die Realien - und hierzu zählen Objekte ebenso wie Textdokumente oder historische Bilddokumente - als Anstöße für historische Vorstellungskraft, die ihrerseits wieder Erinnerungs- und Deutungsleistungen auf den Plan rufen, die in Auseinandersetzung mit 
dem überkommenen historischen Material reflektiert werden müssen. [...] Diese Vorgehensweise ist nicht nur ein Abkömmling der Skepsis, daß eine Gefühlsregung nicht automatisch eine Überzeugung und eine Identifikation noch keine Erkenntnis ist, sondern leitet sich auch von Überlegungen zur Spezifik der nationalsozialistischen Verbrechen, insbesondere des Massenmordes an den europäischen Juden her. [...] Als eine Geschichte lassen sich die NSVerbrechen nur aus einer Perspektive erzählen, was, wenn diese Perspektive die einer Opferoder Widerstandsgruppe ist, als eine besondere Würdeform verstanden werden kann, in geschichtswissenschaftlicher Perspektive aber als Entkontextualisierung bzw. Verkürzung gelten muß.

(Knigge 2002, 385)

Ferner lasse sich die grundlose Auslöschung von Menschen aufgrund ihrer Abstammung nicht ohne eine nachträgliche Sinnkonstruktion erzählen. Knigge tritt hingegen für Ausstellungen ein, die den ,Zivilisationsbruch“ nicht zuschütten: Sie „sehen im Fragmentarischen der Realien gewissermaßen seinen Widerschein und eine Veranlassung für eigenes Suchen, Fragen, Folgern, Stellungnehmen“. (Knigge 2002, 386) Meine Analyse der zehn hier systematisch untersuchten Museen zeigt, dass sich das ,narrative‘ Museum im postsozialistischen Kontext eindeutig durchgesetzt hat - mit Ausnahme von Bulgarien und Rumänien, wie im Abschnitt über missing museums geschildet wird.

Oben bereits angedeutet ist die Verschiebung der Perspektive: 1997 hatte Susan A. Crane den Fokus auf die Opfer und die Berücksichtigung persönlicher Erinnerungen noch als Desiderat eingefordert (Crane 1997, 63), heute ist dies in Gedenkmuseen längst Realität geworden. Märtyrer-, Helden- und Widerstandsnarrative wurden weitestgehend von Opfernarrativen abgelöst. (Rousso 2011, 32; Rahe 2002, 34) Um ausnahmsweise bei einem österreichischen Beispiel zu bleiben: In der Gedenkstätte Mauthausen finden sich auf den alten Gedenktafeln im Lagerbereich noch die traditionellen HeldInnenerzählungen. So heißt es am Eingang zum Quarantänehof: „In diesem Lager wurden über 3000 Frauen verschiedener Nationalität interniert, welche für die Freiheit ihres Landes und den Frieden der ganzen Welt kämpften“, und sogar beim Friedhof der unbekannten Häftlinge steht: „Sie gaben ihr Leben für die Freiheit ihrer Heimat.“ Diese früher völlig unhinterfragte Sinnstiftung steht in einem starken Kontrast zur neuen Museumsausstellung.

Ein Kernpunkt der Untersuchung ist die Frage, ob ,das Opfer' als Individuum oder im Sinne kollektiver Opferschaft Gegenstand der Ausstellungen ist. Individualisierende Opfergeschichten stellen zumeist das gewöhnliche Leben ,davor' aus (Köhr 2007) und ermöglichen Empathie. Besonders wichtig in diesem Zusammenhang sind Ausstellungselemente, in denen die ProtagonistInnen selbst zu Wort kommen. Saul Friedländer betont etwa die Bedeutung von OpferTagebüchern für eine „integrierte Geschichte“ des Holocaust: 
Selbstverständlich muss man die Tagebücher mit derselben kritischen Aufmerksamkeit benutzen wie jedes andere Dokument. Als Quellen für die Geschichte jüdischen Lebens während der Jahre der Verfolgung und Vernichtung bleiben sie jedoch unersetzlich. Hunderte, wahrscheinlich Tausende von Zeugen vertrauten ihre Beobachtungen der Verschwiegenheit ihrer privaten Aufzeichnungen an. Diese Zeugnisse schildern in allen Einzelheiten die Initiativen und die alltägliche Brutalität der Täter, die Reaktionen der Bevölkerung, das Leben und die Vernichtung ihrer Gemeinden, aber sie halten auch die Welt ihres Alltags fest, die von Verzweiflung, Gerüchten, Illusionen und Hoffnung bestimmt ist, welche sich fortwährend abwechseln, meist bis zum Ende.

(Friedländer 2007, 10)

Identifikation mit dem Opfer führt aber keinesfalls automatisch zu Erkenntnis. Als Gründe für die zunehmende Popularisierung des „biographischen Motivs“ von Einzelschicksalen in Holocaustmuseen nennt Köhr $(2012,170)$ den Generationenwechsel, den Einfluss filmischer Erzählungen wie Schindlers Liste oder Der Pianist sowie einen Paradigmenwechsel in der Geschichtswissenschaft von der Sozial- zur Kulturgeschichte. Sie warnt aber vor einer Gefahr der Dekontextualisierung, der Auflösung in zahlreiche Einzelgeschichten, die in vielfältige Zusammenhänge gestellt werden können. (Köhr 2012, 175)

Diese Tendenz wurde etwa bei der Konzeption der 2006 eröffneten Ausstellung in Jasenovac deutlich. Dieses In-situ-Museum plante einen starken Fokus auf die individuellen Opfer, ihre Namen, Hinterlassenschaften und Zeugnisse. Erst im Zuge einer langwierigen Kontroverse konnten Opferverbände und andere inländische KritikerInnen durchsetzen, dass auf einem Bildschirm, auf dem die Namen der bisher namentlich identifizierten knapp 84.000 Opfer durchlaufen (Abb. 2), auch ihr Geburts- und Sterbejahr sowie ihre ethnische Zugehörigkeit angeführt werden. (Radonić 2014b, 96) Das Alter und die ethnische Zuordnung machen nun klar, dass auch Kinder und Greise ermordet wurden, weil sie Serbinnen und Serben, Romnija und Roma oder Jüdinnen und Juden waren, aus ,rassischen Gründen also. Erst dadurch ist gewährleistet, dass das Museum mit der in den 1990er Jahren in Kroatien unter Franjo Tuđman dominanten geschichtsrevisionistischen Deutung der Jasenovac-Opfer als politischen GegnerInnen aufräumt.

Der weitgehende Fokus auf individuelle Opfer birgt die Gefahr der Dekontextualisierung und Enthistorisierung. Die Darstellung des individuellen Opfers als Teil eines nationalen Kollektivopfers, wie sie in anderen Museen anzutreffen ist, verhindert ebenfalls tendenziell Fragen von Mitverantwortung sowie nach dem Verwischen von TäterInnen-, KollaborateurInnen- und Opferrollen und befördert die Externalisierung von Verantwortung an äußere, fremde Mächte, die zu einem „Europa der Opfer“ (Hammerstein und Hofmann 2009, 203; Jureit 2009) führt. „Integrierte Geschichte“ (Friedländer 2007) bezieht hingegen die unverzichtbaren Zeugnisse individueller Opfer ebenso ein wie TäterInnen, KollaborateurInnen und den komplexen historischen Kontext. 


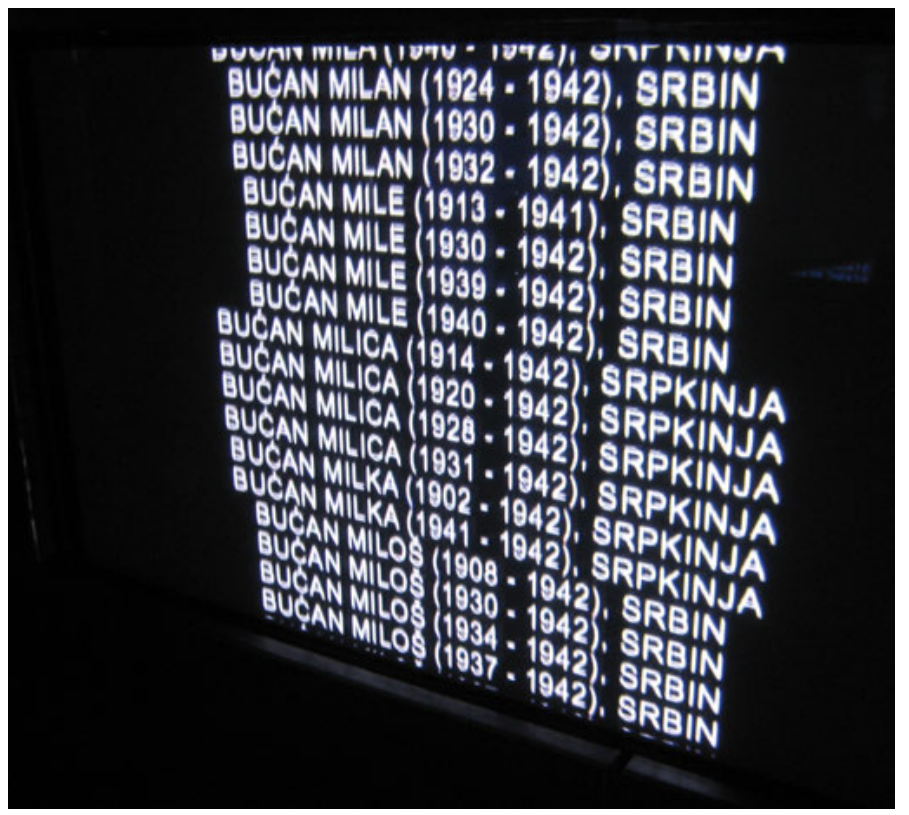

Abb. 2: Auf dem Bildschirm im Jasenovac-Gedenkmuseum laufen die Opferinformationen durch. 